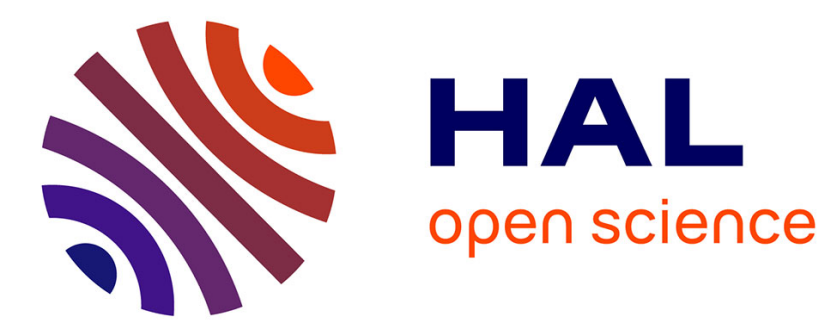

\title{
Image based visual servo-control for a class of aerial robotic systems
}

\author{
Tarek Hamel, Robert Mahony
}

\section{To cite this version:}

Tarek Hamel, Robert Mahony. Image based visual servo-control for a class of aerial robotic systems. Automatica, 2007, 43, pp.1975 - 1983. 10.1016/j.automatica.2007.03.030 . hal-00488389

\section{HAL Id: hal-00488389 \\ https://hal.science/hal-00488389}

Submitted on 1 Jun 2010

HAL is a multi-disciplinary open access archive for the deposit and dissemination of scientific research documents, whether they are published or not. The documents may come from teaching and research institutions in France or abroad, or from public or private research centers.
L'archive ouverte pluridisciplinaire HAL, est destinée au dépôt et à la diffusion de documents scientifiques de niveau recherche, publiés ou non, émanant des établissements d'enseignement et de recherche français ou étrangers, des laboratoires publics ou privés. 


\title{
Image based visual servo-control for a class of aerial robotic systems *
}

\author{
Tarek Hamel $^{\mathrm{a}}$, Robert Mahony ${ }^{\mathrm{b}}$ \\ ${ }^{a}$ I3S UNSA-CNRS, 2000 route des Lucioles - Les Algorithmes - Bt. Euclide B, BP 121, 06903 Sophia Antipolis - cedex France \\ ${ }^{\mathrm{b}}$ Department of Engineering, Australian National University, ACT, 0200, AUSTRALIA.
}

\begin{abstract}
An image-based strategy for visual servo control of a class of dynamic systems is proposed. The class of systems considered includes dynamic models of unmanned aerial vehicles capable of quasi-stationary flight (hover and near hover flight). The control strategy exploits passivity-like properties of the dynamic model to derive a Lyapunov control algorithm using backstepping techniques. The paper extends earlier work, [6], where partial pose information was used in the construction of the visual error. In this paper the visual error is defined purely in terms of the image features derived from the camera input. Local exponential stability of the system is proved. An estimate of the basin of attraction for the closed-loop system is provided.
\end{abstract}

Key words: Image based visual servo, dynamic system, zero dynamics.

\section{Introduction}

Visual servo control concerns the problem of using a camera to provide sensor information to servo-position a robotic system. Classical visual servo control was developed for serial-link robotic manipulators with the camera typically mounted on the end-effector [8]. More recently applications involving mobile systems have been considered [12]. Visual sensing will be a vital technology for the host of low cost unmanned aerial vehicle (UAV) applications that are already under development $[5,16,6]$. Visual servo systems may be divided into two main categories [8]: Pose-Based methods - involving pose reconstruction or estimation and Image-Based methods - working directly with the image data. The first approach is a dual estimation and control problem in which the state (camera pose) of the system is estimated using visual information and the control design is a classical state-space design. The quality of the closed-loop response depends directly on the quality of the pose estimation and makes the control highly sensitive to camera calibration errors and errors in the $3 \mathrm{D}$ model of the target [8]. In the second category, the robotic task is posed in terms of image features rather than in Cartesian

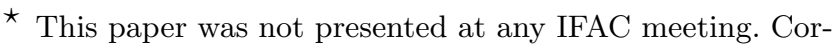
responding author T. Hamel, thamel@i3S.unice.fr.

Email addresses: thamel@i3S.unice.fr (Tarek Hamel), Robert.Mahony@anu.edu.au (Robert Mahony).
}

space. A controller is designed to drive the image features towards a goal configuration that implicitly solves the original Cartesian motion planning problem [4]. The approach is inherently robust to camera calibration and target modelling errors [8].

Classical Image-Based control design uses a simple linearising control for the image kinematics [4] that leads to complex non-linear dynamics and is not easily extended to dynamic system models. Most existing applications exploit a high gain or feedback linearisation (computed torque) design to reduce the system to a controllable kinematic model. There are very few integrated IBVS control designs for fully dynamic system models $[9,18,17,2]$ and even fewer that deal with under-actuated dynamic models. The key problem in applying the classical visual servo control approach lies in the highly coupled form of the image Jacobian. Much of the existing work in visual servo control of UAVs (and particularly autonomous helicopters) have used pose based visual servo methodology $[15,16]$. Prior work by the authors [6] used an image based visual feature augmented with an inertial direction, obtained from a partial attitude poseestimation algorithm.

In this paper, we propose a fully image based visual servo control design for a class of dynamic systems associated with UAV systems capable of quasi-stationary flight. To understand the difficulty of the associated control problem, imagine a UAV (such as a helicopter) hov- 
ering above a fixed target. Imagine that the observed target is slightly to the left of the goal vector in the image. Due to the dynamics of the vehicle, in order to move left the airframe must be inclined to the left, causing the observed landmark to move to the right in the image, while the goal target remains fixed in the image. It follows that to design a stable IBVS control algorithm it is necessary to allow the image error to increase locally in order to allow the system dynamics to act to move the UAV to the desired position such that when the attitude of the UAV is stabilized the image error will be zero. The material presented in the present paper is an extension of our previous work [6] where inertial information was used to augment the goal target in order to avoid the situation just described. In this paper, we deal with the effects of a fixed goal target by treating them as perturbations of the first order image kinematics bounded by error terms that can be derived from the higher order dynamic stabilization problem. A decoupled control design is proposed, followed by a fully coupled stability and robustness analysis. We prove that for a suitable choice of control gains the perturbation in the first order dynamics can be bounded by stability of the attitude dynamics of the system using a structured control Lyapunov function. This provides a robustness guarantee for the resulting control law and leads to excellent simulation results. In this paper, it is assumed that UAV is equipped with an Inertial Measurement Unit (IMU) to compensate gravitational force in the control design and to provide estimates for angular velocity. Additionally, we assume that a measurement of translational velocity is also available. Such a measurement can be obtained from a global position system (GPS) unit, or as a secondary measurement from a vision system.

The paper is organized as follows: Section 2 presents the dynamic system model considered. Section 3 describes the visual features and defines the image based error used. Section 4 derives a Lyapunov control function for the positioning task and an analysis of the stability of the closed-loop system. Section 5 applies the control strategy to a simplified model for the dynamics of a four rotor vertical take-off and landing (VTOL) vehicle known as an X4-flyer [7,1] and presents some simulation results. The final section provides a short summary of conclusions.

\section{Problem Formulation.}

This section presents a dynamic model for the motion of unmanned aerial vehicles capable of stationary hovering at one location. The model used is equivalent to those introduced in the literature to model the dynamics of helicopters $[16,3,7]$. Let $\mathcal{I}=\left\{E_{x}, E_{y}, E_{z}\right\}$ denote the world frame and let $\mathcal{A}=\left\{E_{1}^{a}, E_{2}^{a}, E_{3}^{a}\right\}$ denote the body-fixed frame of the rigid body. The position of the rigid body in the world frame is denoted $\xi=(x, y, z) \in \mathcal{I}$ and its attitude (or orientation) is given by a rotation $R: \mathcal{A} \rightarrow \mathcal{I}$, where $R \in S O(3)$ is an orthogonal rotation matrix. Let
$V$ (resp. $\Omega$ ) denote the translational (resp. angular) velocity of the body expressed in the body fixed frame. Let $m$ denote the total mass and $\mathbf{I}=\operatorname{diag}\left(\mathbf{I}_{1}, \mathbf{I}_{2}, \mathbf{I}_{3}\right)$ a diagonal matrix denoting the inertia of the body. The dynamics of a rigid body are $^{1}$ :

$$
\begin{aligned}
\dot{\xi} & =R V \\
m \dot{V} & =-m \Omega \times V+F=-m \operatorname{sk}(\Omega) V+F \\
\dot{R} & =R \operatorname{sk}(\Omega), \\
\mathbf{I} \dot{\Omega} & =-\Omega \times \mathbf{I} \Omega+\Gamma=-\operatorname{sk}(\Omega) \mathbf{I} \Omega+\Gamma .
\end{aligned}
$$

The exogenous force and torque are denoted $F$ and $\Gamma$ respectively (cf. Fig. 1). The inputs considered corre-

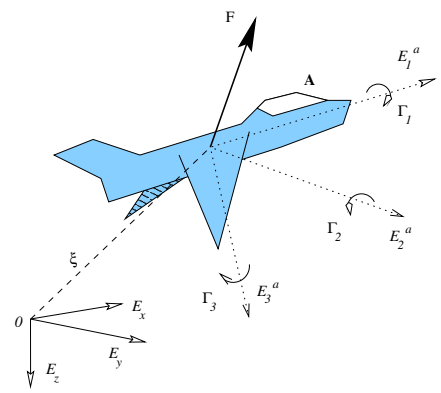

Fig. 1. Reference frames, forces and torques for an Unmanned Aerial Vehicle (UAV).

spond to a typical arrangement found on a VTOL aircraft (cf. Sec. 5). The inputs are written as a single translational force, denoted $F$ in Figure 1, along with full torque control, shown by the torques $\Gamma_{1}, \Gamma_{2}$ and $\Gamma_{3}$ around axes $E_{1}^{a}, E_{2}^{a}$ and $E_{3}^{a}$ respectively. The force $F$ combines thrust, lift, gravity and drag components. It is convenient to separate the gravity component $m g E_{z}=$ $m g R^{T} e_{3}$ from the combined aerodynamic forces and assume that the aerodynamic forces are always aligned with the $z$-axis in the body fixed frame ${ }^{2}$,

$$
F:=-T e_{3}+m g R^{T} e_{3}
$$

where $T \in \mathbb{R}$ is a scalar input representing the magnitude of external force applied in direction $e_{3}$. Control of the airframe is obtained by using the torque control $\Gamma=$ $\left(\Gamma_{1}, \Gamma_{2}, \Gamma_{3}\right)$ to align the force $F_{0}:=T e_{3}$ as required to track the goal trajectory.

\section{Image feature and dynamics}

Classical visual servo control schemes choose sufficient image features to control all six degrees of freedom of

\footnotetext{
1 The notation $\operatorname{sk}(\Omega)$ denotes the skew-symmetric matrix such that $\operatorname{sk}(\Omega) v=\Omega \times v$ for the vector cross-product $\times$ and any vector $v \in \mathbb{R}^{3}$.

2 This is a reasonable assumption for the dynamics of a UAV in quasi-stationary flight where the exogenous force is dominated by the lift force while drag and forward thrust are negligible.
} 
the camera frame of reference $[4,8]$. This cannot be done for the control of an under-actuated system as the dynamic constraints between states will prevent certain configurations from being stabilisable. For the model class considered there are four independent inputs $\left(T, \Gamma_{1}, \Gamma_{2}, \Gamma_{3}\right)$ for the six degrees of the freedom of the system $(x, y, z, \phi, \theta, \psi)$, where $(\phi, \theta, \psi)$ denote the yaw-pitch-roll Euler angles. In principal, it is possible to stabilise four independent outputs, however, the dynamic constraints of the system prevent arbitrary input-output assignment. The equilibrium condition for the dynamics of the translational motion (Eq. 2) implies that

$$
\dot{V}_{\infty}=V_{\infty}=F_{\infty}=0 .
$$

Substituting into Eq. 5 one has

$$
R_{\infty}^{T} e_{3}=e_{3}, \quad T_{\infty}=m g
$$

It follows that the asymptotic values of the pitch $\left(\theta_{\infty}\right)$ and roll $\left(\psi_{\infty}\right)$ are fixed by the stability condition. Conversely, the remaining four independent state variables $(x, y, z, \phi)$ can be assigned an arbitrary set point. To pose a well defined visual servo control task it is necessary to find visual outputs for the partial state $(x, y, z, \phi)$ that are independent of the states $(\theta, \psi)$. In practice, the stabilization of $\phi$ involves a significant level of additional complexity in the control design that has little to do with the principal issues addressed in this paper. For this reason we will consider a quasi-equilibrium in the yaw direction corresponding to the output states

$$
Y_{\text {out }}=(x, y, z, \dot{\phi}) \text {. }
$$

In visual servo control problems, the image dynamics must be expressed in the frame of reference of the camera. Since we consider a camera mounted on the vehicle, the motion of the camera frame inherits dynamics in the body fixed frame. In order to simplify the derivation in the sequel, it is assumed that the camera fixed frame coincides with the body fixed frame $\mathcal{A}$. Let $P_{i}^{\prime}$ denote a set of $n$ points of a stationary target in the inertial frame $(\mathcal{I})$. Let $P_{i}$ be the same points expressed in camera-fixed-frame

$$
P_{i}=R^{T}\left(P_{i}^{\prime}-\xi\right)
$$

Backstepping control design has passivity-like properties from virtual input to the backstepping error [11]. In our previous work [6] it was shown that these structural passivity-like properties are present in the image space dynamics if and only if the spherical projection of an observed point is used (see [6] for additional details). Denoting the spherical projection of an image point $P_{i}$ by $p_{i}$ the image dynamics are given by:

$$
\dot{p}_{i}=-\operatorname{sk}(\Omega) p_{i}-\pi_{p_{i}} \frac{V_{i}}{r_{i}}
$$

Here $r_{i}=\left|P_{i}\right|$ (the focal length is assumed to be unity for simplicity) and $V_{i} \in \mathcal{A}$ is the velocity of the target point represented in the camera fixed frame. The matrix $\pi_{p_{i}}=\left(I_{3}-p_{i} p_{i}^{T}\right)$ is the projection onto the tangent space of the spherical image surface at the point $p_{i}\left(I_{3}\right.$ is the $3 \times 3$ identity matrix).

If the inertial velocity of the target point $P_{i}^{\prime}$ is equal to zero then $V_{i}=-V$. In this case, Eq. 8 becomes

$$
\dot{p}_{i}=-\operatorname{sk}(\Omega) p_{i}-\frac{\pi_{p_{i}}}{r_{i}} V
$$

For a given target the centroid of the target based on spherical information is defined to be

$$
q:=\sum_{i=1}^{n} p_{i} \in \mathbb{R}^{3}
$$

The three entries in the feature $q$ provide information corresponding to position $(x, y, z)$ in the camera-fixedframe. The depth information, that is the key problem in most visual servo algorithms, is in one-to-one correspondence with a non-linear function of the norm $|q|$ [14]. Direct information on the inertial position cannot be obtained without an estimate of the orientation, $R$, of the vehicle. The final degree of freedom considered $\dot{\phi}$ does not required a visual error as it can be measured using gyros.

Recalling Eq. 9, it may be verified that

$$
\dot{q}=-\operatorname{sk}(\Omega) q-Q V
$$

where

$$
Q=\sum_{i=1}^{i=n} \frac{\pi_{p_{i}}}{r\left(P_{i}\right)} .
$$

The visual servo control task considered is that of positioning a camera relative to a stationary (in the inertial frame) target. In our previous work [6], in addition to visual information, inertial information is explicitly used in the error formulation. In particular, the inertial direction of the goal target in the image is fixed. That is, a goal $q^{*}$ was chosen that had fixed inertial orientation and inherited the ego motion of the camera, $\dot{q}^{*}=-\operatorname{sk}(\Omega) q^{*}$. The advantage of such a choice is clear when one recalls the example mentioned in the introduction of a UAV (such as a helicopter) hovering above a fixed target. If the image vector has fixed inertial direction, then as the UAV tilts the image error does not change. The goal vector $q^{*}(t)$ moves in the (spherical) image plane in exactly the same manner as the observed target. It is only as the vehicles changes its position that the image error changes. In prior work [6], this property was exploited to decouple the position stabilization from the attitude stabilization problem.

If no inertial information for the goal vector is known 
then we propose to define a fixed goal vector and deal with coupled dynamic equations. Define $q^{*} \in \mathcal{A}$ to be an attainable desired target vector expressed in the camera fixed frame.

$$
q^{*}:=\left(q_{1}^{*}, q_{2}^{*}, q_{3}^{*}\right)^{T} \in \mathcal{A}
$$

The target vector does not inherit the ego motion of the camera, $\dot{q}^{*}=0$. The norm $\left|q^{*}\right|$ encodes the effective depth information for the desired limit point while the direction of $q^{*}$ defines the camera attitude up to rotation around the axis $q^{*}$ in the desired limit configuration.

The image based error considered is the difference between the measured centroid and the target vector expressed in the camera fixed frame

$$
\delta_{1}:=q-q^{*} .
$$

Deriving $\delta_{1}$ yields:

$$
\dot{\delta}_{1}=-\operatorname{sk}(\Omega) \delta_{1}-Q V-\operatorname{sk}(\Omega) q^{*}
$$

The above equation (Eq. 15) defines the kinematics of the visual error $\delta_{1}$.

It is of interest to study the structural properties of Eq. 15. Consider the storage function $\left|\delta_{1}\right|^{2}$. The derivative of this function is

$$
\frac{d}{d t}\left|\delta_{1}\right|^{2}=-\delta_{1}^{T} \operatorname{sk}(\Omega) \delta_{1}-\delta_{1}^{T} Q V-\delta_{1}^{T} \operatorname{sk}(\Omega) q^{*} .
$$

The first term is zero due to the skew symmetry of $\Omega$. Since the matrix $Q>0$ is positive definite, the second term can be seen as an inner product between $\delta_{1}$ and $V$. In this sense we think of the second term as a the supply function to the storage function. Choosing $V=\delta_{1}$ acts to decrease $\left|\delta_{1}\right|^{2}$. The last term is the perturbation due to the fixed goal assumption and was not present in the prior work of the authors [6]. When the goal vector is fixed in the image plane it is impossible to avoid the presence of the perturbation term. The term resembles the perturbations due to small-body forces that lead to zero dynamic effects studied in recent work $[5,13]$. This sort of term is particularly difficult to deal with explicitly in the control design. It depends on the angular velocity $\Omega$ and destroys the pure feedback nature of the system that is necessary to apply the backstepping approach. The approach taken in this paper is to leave this term as a perturbation of the stability analysis of the first order image kinematics. By choosing the control gains governing the higher order backstepping errors correctly this error perturbation can be controlled and dominated in the integrated stability analysis.

\section{Visual servo control for a VTOL aircraft}

This section presents a backstepping control design for visual servo control of the under-actuated system con- sidered. The full dynamics of the error $\delta_{1}$ may be written

$$
\begin{aligned}
\dot{\delta}_{1} & =-\operatorname{sk}(\Omega) \delta_{1}-Q V-\operatorname{sk}(\Omega) q^{*} \\
m \dot{V} & =-m \operatorname{sk}(\Omega) V+F \\
\dot{R} & =R \operatorname{sk}(\Omega), \\
\mathbf{I} \dot{\Omega} & =-\operatorname{sk}(\Omega) \mathbf{I} \Omega+\Gamma .
\end{aligned}
$$

Before introducing the main result of the paper, we introduce the following change of variables:

$$
\begin{aligned}
& \delta_{2}=\frac{m}{k_{1}} V-\delta_{1}, \\
& \delta_{3}=\frac{m}{k_{1}^{2} k_{2}}\left(-T e_{3}+m g R^{T} e_{3}\right)+\delta_{2}
\end{aligned}
$$

The error $\delta_{2}$ is introduced to regulate the translational velocity of the camera and ensures that it comes to rest. The additional error vector $\delta_{3}$ incorporates information on the attitude of the camera. This is natural for a system such a VTOL aircraft capable of quasi-stationary flight, since the desired motion can only be obtained by exploiting the attitude dynamics to regulate $\delta_{1}$. If the position and translational velocity are regulated then the total external force must be zero, $-T e_{3}+m g R^{T} e_{3}=0$. Recalling Eq. 6 one has $R e_{3}=e_{3}$ and $T=m g$. This implies that the pitch and roll rotations of the rigid body are directly stabilised via the stabilisation of the error $\delta_{3}$. This corresponds to the input-output structure discussed in Section 3. As expected, the yaw rotation around the direction $e_{3}$ is independent of the error $\delta_{3}$. The remaining output state considered is the yaw rate $\dot{\phi}=\Omega_{3}$. To simplify the remainder of the development we will assume that this state is in equilibrium at time zero, $\Omega_{3}(0)=0$. A simple linearising proportional control is applied in the third component of angular velocity to maintain the condition

$$
\Gamma_{3}=e_{3}^{T}(\operatorname{sk}(\Omega) \mathbf{I} \Omega)-k_{\Omega} \Omega_{3}
$$

where $k_{\Omega}$ is a suitable positive gain. In practice, the above control will quickly stabilise the system from nonzero initial conditions. In the sequel we will assume that $\Omega_{3}(0)=0$ and therefore $\Omega_{3}(t)=0$, for all $t>0$.

The proposed control algorithm requires a formal time derivative of the input $T$. To provide this input, $T$ is dynamically extended

$$
\dot{T}=U
$$

The motivation for adding a differentiator is to ensure decoupling between translational and rotational dynamics as shown in the sequel.

Recalling Eqn's 16-27, the full dynamics of the visual error $\delta_{1}$ in terms of the additional errors $\delta_{i}(i=1 \ldots 3)$ 
can be written

$$
\begin{gathered}
\dot{\delta}_{1}=-\operatorname{sk}(\Omega) \delta_{1}-\frac{k_{1}}{m} Q \delta_{1}-\frac{k_{1}}{m} Q \delta_{2}+\operatorname{sk}\left(q^{*}\right) \Omega \\
\dot{\delta}_{2}=-\operatorname{sk}(\Omega) \delta_{2}+\frac{k_{1}}{m} Q \delta_{1}-\frac{k_{1}}{m}\left(k_{2} I-Q\right) \delta_{2}+\frac{k_{1} k_{2}}{m} \delta_{3} \\
\quad-\operatorname{sk}\left(q^{*}\right) \Omega \\
\dot{\delta}_{3}=-\operatorname{sk}(\Omega) \delta_{3}+\frac{k_{1}}{m} Q \delta_{1}-\frac{k_{1}}{m}\left(k_{2} I-Q\right) \delta_{2}+\frac{k_{1} k_{2}}{m} \delta_{3} \\
+\frac{m}{k_{1}^{2} k_{2}}\left(\operatorname{sk}\left(T e_{3}-\frac{k_{1}^{2} k_{2}}{m} q^{*}\right) \Omega-\dot{T} e_{3}\right)
\end{gathered}
$$

Define $\delta_{4}$ as a final error term. It will regulate the pitch and roll angular velocities as

$\delta_{4}=\frac{m^{2}}{k_{1}^{2} k_{2}\left(k_{1} k_{2}+k_{3}\right)} \pi_{e_{3}} \operatorname{sk}\left(T e_{3}-\frac{k_{1}^{2} k_{2}}{m} q^{*}\right) \Omega+\pi_{e_{3}} \delta_{3}$,

where $\pi_{e_{3}}=\left(I-e_{3} e_{3}^{T}\right)$. Using the assumption $\Omega_{3}=$ 0 (cf. Eq. ??) and introducing Eqn's 23 and 27 in the expression of the derivative of error $\delta_{3}$ (Eq. 26) yields

$$
\begin{aligned}
\dot{\delta}_{3} & =-\operatorname{sk}(\Omega) \delta_{3}+\frac{k_{1}}{m} Q \delta_{1}-\frac{k_{1}}{m}\left(k_{2} I-Q\right) \delta_{2} \\
& +\frac{k_{1} k_{2}+k_{3}}{m} \pi_{e_{3}} \delta_{4}-\frac{k_{3}}{m} \delta_{3} \\
& -\left(I-\pi_{e_{3}}\right)\left(\operatorname{sk}\left(q^{*}\right) \pi_{e_{3}} \Omega-\frac{k_{1} k_{2}+k_{3}}{m} \delta_{3}+\frac{m}{k_{1}^{2} k_{2}} U e_{3}\right)
\end{aligned}
$$

Deriving the expression of $\delta_{4}$ (Eq. 27) and recalling Eqn's $24,25,23$ and 28 , it yields

$$
\begin{aligned}
\dot{\delta}_{4} & =-\pi_{e_{3}} \operatorname{sk}(\Omega) \delta_{3}+\frac{k_{1}}{m} \pi_{e_{3}} Q \delta_{1}-\frac{k_{1}}{m} \pi_{e_{3}}\left(k_{2} I-Q\right) \delta_{2} \\
& +\frac{k_{1} k_{2}+k_{3}}{m} \pi_{e_{3}} \delta_{4}-\frac{k_{3}}{m} \pi_{e_{3}} \delta_{3} \\
& +\frac{m^{2}}{k_{1}^{2} k_{2}\left(k_{1} k_{2}+k_{3}\right)} \pi_{e_{3}} U \operatorname{sk}\left(e_{3}\right) \pi_{e_{3}} \Omega \\
& +\frac{m^{2}}{k_{1}^{2} k_{2}\left(k_{1} k_{2}+k_{3}\right)} \pi_{e_{3}} \operatorname{sk}\left(T e_{3}-\frac{k_{1}^{2} k_{2}}{m} q^{*}\right) \pi_{e_{3}} \dot{\Omega}
\end{aligned}
$$

It is now possible to present the main result of the paper.

Theorem 1 Consider the dynamics given by Eqn's 24, 25, 28 and 29. Let $\lambda_{\max }>0$ be a bound on the 2-norm of the matrix $Q$

$$
\|Q\|_{2} \leq \lambda_{\max }
$$

Let the vector controller be given by

$$
\begin{gathered}
U=\frac{k_{1}^{2} k_{2}}{m} \frac{k_{1} k_{2}+k_{3}}{m} e_{3}^{T} \delta_{3}-\frac{k_{1}^{2} k_{2}}{m} e_{3}^{T} \operatorname{sk}\left(q^{*}\right) \Omega \\
\Gamma=\mathbf{I}^{-1}\left(\operatorname{sk}(\Omega) \mathbf{I} \Omega-k_{\Omega} \Omega_{3} e_{3}-\frac{\mathbf{I}}{T-\frac{k_{1}^{2} k_{2}}{m} q_{3}^{*}} U \operatorname{sk}\left(e_{3}\right) \Omega\right. \\
-\frac{k_{1}^{2} k_{2}\left(k_{1} k_{2}+k_{3}\right)}{\left(T-\frac{k_{1}^{2} k_{2}}{m} q_{3}^{*}\right) m^{2}} \operatorname{sk}\left(e_{3}\right)\left(\operatorname{sk}(\Omega) \delta_{3}+\frac{k_{1} k_{2}}{m} \delta_{2}-\frac{k_{1} k_{2}}{m} \delta_{3}\right. \\
\left.\left.-\frac{k_{1} k_{2}+k_{3}+k_{4}}{m} \delta_{4}\right)\right)
\end{gathered}
$$

Define a storage function for the visual scheme by

$$
\mathcal{L}=\frac{1}{2}\left|\delta_{1}\right|^{2}+\frac{1}{2}\left|\delta_{2}\right|^{2}+\frac{1}{2}\left|\delta_{3}\right|^{2}+\frac{1}{2}\left|\delta_{4}\right|^{2}
$$

Set

$$
f(T)=\frac{k_{1} k_{2}\left(k_{1} k_{2}+k_{3}\right)}{m\left(T-\frac{k_{1}^{2} k_{2}}{m} q_{3}^{*}\right)}
$$

and choose the positive control gains $\left(k_{1}, \ldots, k_{4}\right)$ to satisfy

$$
\begin{aligned}
& k_{2}>\lambda_{\max }(Q), \\
& k_{3}>\frac{k_{1}\left(k_{2}\left(\lambda_{\max }+2 f\left(T_{\min }\right)\left|q^{*}\right|\right)+2 f\left(T_{\min }\right)\left|q^{*}\right|^{2}\right)}{\left(k_{2}-\lambda_{\max }\right)},
\end{aligned}
$$

where $T_{\min }=2 \frac{k_{1}^{2} k_{2}}{m} q_{3}^{*}$. Then, for any initial condition such that $\Omega_{3}(0) \stackrel{m}{=} 0$ and that the initial value of the Lyapunov function satisfies

$$
\mathcal{L}(0)<\frac{1}{2}\left(\frac{m^{2} g}{k_{1}^{2} k_{2}}-2 q_{3}^{*}\right)^{2},
$$

$\mathcal{L}(t)$ is exponentially decreasing. The error coordinates $\left(\delta_{1}, \ldots, \delta_{4}\right)$ converge exponentially to zero. The closedloop system is locally exponentially stable.

Proof Recall that

$$
\dot{\Omega}=-\mathbf{I}^{-1} \operatorname{sk}(\Omega) \mathbf{I} \Omega+\mathbf{I}^{-1} \Gamma
$$

Note that

$$
\pi_{e_{3}} \operatorname{sk}\left(T e_{3}-\frac{k_{1}^{2} k_{2}}{m} q^{*}\right) \pi_{e_{3}}=\left(T-\frac{k_{1}^{2} k_{2}}{m} q_{3}^{*}\right) \operatorname{sk}\left(e_{3}\right)
$$

and that $\pi_{e_{3}} \delta_{4}=\delta_{4}$. Differentiating $\mathcal{L}$ and substituting from Eqn's 24-25, 28-29 along with the feedback control 
(Eqn's 30 and 31) one obtains

$$
\begin{aligned}
\dot{\mathcal{L}} & =-\frac{k_{1}}{m} \delta_{1}^{T} Q \delta_{1}-\frac{k_{1}}{m} \delta_{2}^{T}\left(k_{2} I_{3}-Q\right) \delta_{2}-\frac{k_{3}}{m}\left|\delta_{3}\right|^{2}-\frac{k_{4}}{m}\left|\delta_{4}\right|^{2} \\
& +\frac{k_{1}}{m} \delta_{3}^{T} Q \delta_{1}+\frac{k_{1}}{m} \delta_{3}^{T} Q \delta_{2}+\frac{k_{1}}{m} \delta_{4}^{T} Q \delta_{1}+\frac{k_{1}}{m} \delta_{4}^{T} Q \delta_{2} \\
& +\left(\delta_{1}-\delta_{2}\right)^{T} \operatorname{sk}\left(q^{*}\right) \Omega
\end{aligned}
$$

Note that, due to the dependence on the angular velocity $\Omega$ it is not immediately clear that the storage function is decreasing. Recall the expression of the error term $\delta_{4}$ Eq. 27. One has

$$
\pi_{e_{3}} \Omega=-\frac{k_{1}}{m} f(T) \operatorname{sk}\left(e_{3}\right)\left(\delta_{4}-\delta_{3}\right)
$$

Substituting for $\Omega$ in Eq. 34 one obtains

$$
\dot{\mathcal{L}} \leq-\Delta^{T} \Sigma \Delta
$$

where:

$$
\Delta=\left(\begin{array}{l}
\delta_{1} \\
\delta_{2} \\
\delta_{3} \\
\delta_{4}
\end{array}\right), \Sigma=\left(\begin{array}{cccc}
k_{1} Q & 0 & -\frac{k_{1}}{2} M & -\frac{k_{1}}{2} N \\
0 & k_{1}\left(k_{2} I-Q\right) & -\frac{k_{1}}{2} N & -\frac{k_{1}}{2} M \\
-\frac{k_{1}}{2} M & -\frac{k_{1}}{2} N & k_{3} & 0 \\
-\frac{k_{1}}{2} N & -\frac{k_{1}}{2} M & 0 & k_{4}
\end{array}\right),
$$

$-\operatorname{sk}\left(q^{*}\right) \mathrm{sk}\left(e_{3}\right)$. The above quadratic expression is negative definite if and only if the symmetric matrix $\Sigma$ is positive definite. This is true if and only if the principal minors of $\Sigma$ are positive. This yields the following sufficient conditions on the positive control gains $\left(k_{1}, \ldots, k_{4}\right)$ :

$k_{2}>\lambda_{\max }$

$k_{3}>\frac{k_{1}\left(k_{2}\left(\lambda_{\max }+2 f\left(T_{\min }\right)\|A\|\right)+2 f\left(T_{\min }\right)\left\|A A^{T}\right\|^{2}\right)}{\left(k_{2}-\lambda_{\max }\right)}$,

where $\|\cdot\|$ denotes the matrix Frobenius matrix norm. In order to derive the conditions provided by the theorem statement insuring the definite negativeness of the storage function $\mathcal{L}$ and that the control law Eq. 31 is well defined all the time well, in particular, that $T-\frac{k_{1}^{2} k_{2}}{m} q_{3}^{*}$ does not vanish, the following two sided bound on the control $T=T(t)$ is obtained from Eq. 21

$m g-\frac{k_{1}^{2} k_{2}}{m}\left(\left|\delta_{2}\right|+\left|\delta_{3}\right|\right)<T(t)<m g+\frac{k_{1}^{2} k_{2}}{m}\left(\left|\delta_{2}\right|+\left|\delta_{3}\right|\right)$.

Combining this bound with the expression for the storage function, one obtains

$$
m g-\frac{k_{1}^{2} k_{2}}{m} \sqrt{2 \mathcal{L}}<T(t)<m g+\frac{k_{1}^{2} k_{2}}{m} \sqrt{2 \mathcal{L}}
$$

ensures that for all $\forall t>0, T(t)>T_{\min }$. It follow that the control law Eq. 31 is well defined for all time and guarantees that the derivative of the storage function is negative definite.

The uniqueness of the control input is guaranteed as long as $T(t) \neq \frac{k_{1}^{2} k_{2}}{m}\left|q_{3}^{*}\right|$ (cf. Eq. 31).

Combining the above inequality with constraint on the initial conditions Eq. 33 of the theorem yields the following bound on the initial condition of the control $T$

$$
\begin{aligned}
2 \frac{k_{1}^{2} k_{2}}{m}\left|q_{3}^{*}\right|< & \left(m g-\frac{k_{1}^{2} k_{2}}{m} \sqrt{2 \mathcal{L}(0)}\right) \\
& <T(0)<\left(m g+\frac{k_{1}^{2} k_{2}}{m} \sqrt{2 \mathcal{L}(0)}\right)
\end{aligned}
$$

It remains to show that this inequality is verified for all $t>0$. To this end, we will assume first that $T(t)>$ $T_{\min }=2 \frac{k_{1}^{2} k_{2}}{m}\left|q_{3}^{*}\right|, \forall t>0$. In this situation, the choice of gains $k_{1}, \ldots, k_{4}$ ensures the positiveness of the principal minors of the matrix $\Sigma$ and therefore the definite negativeness of the storage function derivative. Combining both above inequalities, it follows that

$$
\begin{aligned}
2 \frac{k_{1}^{2} k_{2}}{m}\left|q_{3}^{*}\right|< & \left(m g-\frac{k_{1}^{2} k_{2}}{m} \sqrt{2 \mathcal{L}(t)}\right) \\
& <T(t)<\left(m g+\frac{k_{1}^{2} k_{2}}{m} \sqrt{2 \mathcal{L}(t)}\right)
\end{aligned}
$$

Finally, the analysis has shown that $\mathcal{L}(t)$ is exponentially decreasing for suitable initial conditions and the result follows from Lyapunov's direct method [10].

Theorem 1 proves only local exponential convergence. The saturation constraints on the control input $T$ suggests that it is impossible to extend the result to global exponential stability. It is of interest, however, to obtain some information on the basin on attraction of the system and suitable choice of gains to ensure stability of the closed-loop system. Consider initial conditions where the system is in stationary flight. That is

$$
V(0)=0, \quad \Omega(0)=0, \quad T(0) e_{3}=m g R(0)^{T} e_{3} .
$$

As a consequence of this choice one has

$$
\delta_{2}(0)=\delta_{1}(0), \quad \delta_{3}(0)=\delta_{1}(0), \quad \delta_{4}(0)=\pi_{e_{3}} \delta_{1}(0)
$$

It is straightforward to see that $\mathcal{L}<2|\delta(0)|^{2}$ under these conditions. Thus, the condition Eq. 33 in Theorem 1 is guaranteed if

$$
2 \delta_{1}^{2}(0)<\frac{1}{2}\left(\frac{m^{2} g}{k_{1}^{2} k_{2}}-2 q_{3}^{*}\right)^{2}
$$


Equation 36 has a natural physical interpretation; if the gain $k_{1}$ for stability of the image feature $\delta_{1}$ is sufficiently small then the demanded motion of the system will be slow and the closed-loop response will not excite the unmodeled zero dynamics of the system, ensuring that the stability analysis is valid. It is interesting to note that $\delta_{1}$ is naturally saturated due to the nature of the discrete centroid image feature used as shown in simulation section 5 .

Corollary 1 Consider the same conditions as given for Theorem 1. The state $(\xi, R, V, \Omega)$ converges exponentially to a point $\left(\xi_{\infty}, R_{\infty}, 0,0\right)$ such that

$$
R_{\infty}=\left(\begin{array}{ccc}
\cos \phi_{\infty} & -\sin \phi_{\infty} & 0 \\
\sin \phi_{\infty} & \cos \phi_{\infty} & 0 \\
0 & 0 & 1
\end{array}\right) .
$$

If $q^{*}=\left|q^{*}\right| e_{3}$ is collinear with the direction of gravity then $\xi_{\infty}=-\alpha e_{3}$, for some $\alpha>0$, is an isolated point. If $q^{*} \times e_{3} \neq 0$ then there is a smooth set of exponentially stable $\xi_{\infty}$ parameterized by $\phi_{\infty}$.

Proof Theorem 1 ensures that $\delta_{i} \rightarrow 0$ exponentially. Setting $\delta_{i}=0$ and recalling Eqn's 7-10 and 13, one obtains

$$
R_{\infty}^{T} q_{\infty}^{0}=q^{*}
$$

where $q_{\infty}^{0}$ represents the inertial representation of $q^{*}$. In addition, one has that $V_{\infty}=\Omega_{\infty}=0$ and (from Eq. 21), the total external force must be zero, $-T_{\infty} e_{3}+$ $m g R_{\infty}^{T} e_{3}=0$. It follows that $R_{\infty} e_{3}=e_{3}$ and the pitch and roll angles are directly stabilized via the regulation of $\delta_{1}$. The yaw angular velocity is exponentially stable and the rotation matrix must converge exponentially to the form of Eq. 37 for some $\phi_{\infty}$ that depends on initial conditions.

If $q^{*}=\left|q^{*}\right| e_{3}$ then one has that $q_{\infty}^{0}=q^{*}=\left|q^{*}\right| e_{3}$. By construction of the inertial frame this implies that $\xi_{\infty}$ must also be collinear with $e_{3}$. The first result follows directly.

If $q^{*} \times e_{3} \neq 0$ then $R_{\infty}^{T} q_{\infty}^{0}=q^{*}$ induces a relationship between the angle $\phi_{\infty}$ and the value of $q_{\infty}^{0}$. It is straightforward to see that this relationship is non-degenerate, that is each $\phi_{\infty}$ leads to a unique $q_{\infty}^{0}$ and consequently $\xi_{\infty}$. The exact relationship depends on the geometry of the target constellation and cannot be easily characterized.

If the control design is extended to stabilize the yaw angle instead of the yaw angle velocity [6] then the limit point $\left(\xi_{\infty}, R_{\infty}, 0,0\right)$ is the unique set point for the system.

\section{Example System and Simulation}

In this section, the procedure presented in Section 4 is applied to an idealised model of the dynamics of an X4flyer. This flying robot consists of four individual fans fixed to a rigid cross frame. It operates as an omnidirectional vehicle capable of quasi-stationary flight. An idealised dynamic model of the X4-flyer $[7,1]$ is given by the rigid body equations (Eqn's 1-4) along with the external force and torque inputs (cf. Fig. 2)

$$
\begin{aligned}
T & =T_{r r}+T_{r l}+T_{f r}+T_{f l}, \\
\Gamma_{1} & =d\left(T_{f r}+T_{f l}-T_{r r}-T_{r l}\right), \\
\Gamma_{2} & =d\left(T_{r l}+T_{f l}-T_{r r}-T_{f r}\right), \\
\Gamma_{3} & =Q\left(T_{f r}\right)+Q\left(T_{r l}\right)+Q\left(T_{f l}\right)+Q\left(T_{r r}\right) \\
& =\kappa\left(T_{f r}+T_{r l}-T_{f l}-T_{r r}\right) .
\end{aligned}
$$

The individual thrust of each motor is denoted $T_{(.)}$, while $\kappa$ is the proportional constant giving the induced couple due to air resistance for each rotor and $d$ denotes the distance of each rotor from the centre of mass of the X4flyer. The parameters used for the dynamic model are

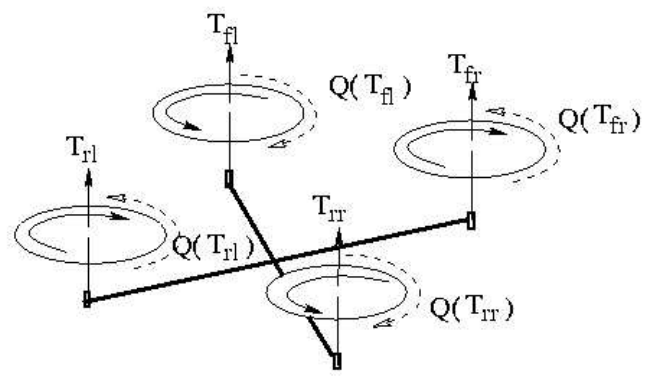

Fig. 2. The force and torque inputs for an X4-flyer.

$m=1 \mathrm{~kg}, I=\operatorname{diag}(0.42,0.42,0.22) \mathrm{kg} \cdot \mathrm{m}, d=0.25 \mathrm{~m}$, $\kappa=0.01 \mathrm{~m}$ and $g=9.8 \mathrm{~m} . \mathrm{s}^{-2}$.

The simulation undertaken considers the case of stabilisation of the X4-flyer, initially in hover flight, to a new set point several metres distance from the initial condition. Consider the case where one wishes to position the camera parallel to a plan target characterised by a square. The vertex length is of 2 meters. In the case of a pin-hole camera the visual measurements available are the projective coordinates of the four points defining the square. Re-normalising the visual data during calibration, we assume without loss of generality that the focal length of the camera is unity and the focal plane is spherical. The desired target vector $q^{*}$ is chosen such that the camera set point is located 3 metres above the square. Using the above specification, the desired feature $q^{*}=(0,0,3.6181)^{T}$. For the simulation undertaken, the bound $\lambda_{\max }=2 \mathrm{~m}^{-1}$ was used. The initial 
condition was chosen such that the X4-flyer was in stable stationary flight $\left(V_{0}=\Omega_{0}=0\right.$ and $\left.T_{0}=g=9.8\right)$ with $\xi_{0}=(-1.5-2.5-4)^{T 3}$ and $R_{0}=I_{3}$. The control gains used were $k_{1}=0.35 \mathrm{~kg} . \mathrm{m} . \mathrm{s}^{-1}, k_{2}=6 \mathrm{~kg}^{-1}$, $k_{3}=65 \mathrm{~kg} \cdot \mathrm{s}^{-1}, k_{4}=3 \mathrm{~kg} . \mathrm{s}^{-1}$. These gains ensure that the condition Eq. 36 holds,

$$
2\left|\delta_{1}(0)\right|^{2}=10.2514<18.5875=\frac{1}{2}\left(\frac{m g}{k_{1}^{2} k_{2}}-2 q_{3}^{*}\right)^{2},
$$

and consequently that the initial conditions satisfy the conditions of Theorem 1 (Eq. 33). This bound $2\left|\delta_{1}(0)\right|^{2}<18.5875$ provides an inner bound on the size of the basin of attraction for the closed-loop system. Figure 3 plots a slice through the shape of the basin (in the Cartesian space) for the example considered. Note that the basin extends infinitely high above the target, this is to be expected due to the bounded magnitude of the image feature. The inner bound on the basin shape is generated by the constraint on $\|Q\|_{2} \leq \lambda_{\max }$. It is clear that the overall shape of the basin of attraction is reminiscent of a conic section (cut off close to the target). By decreasing $k_{1}$ the slope of the edge of the basin is flattened and the basin enlarged in a natural manner.

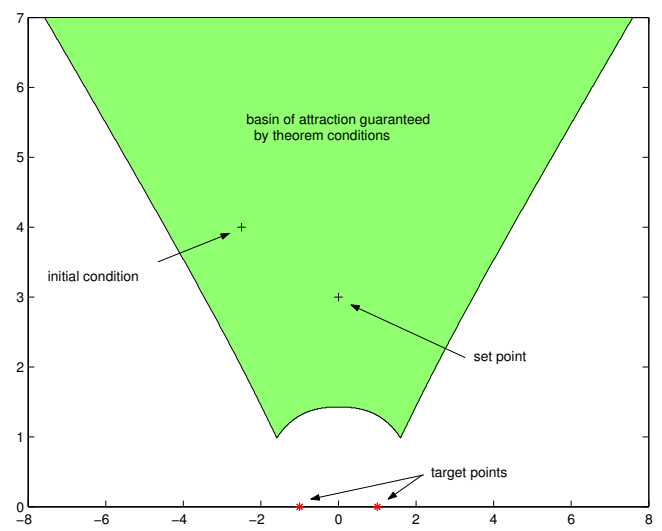

Fig. 3. An inner bound on basin of attraction computed from the bound Eq. 36 for initial conditions where the device is in stable hover. The present plot displays a slice through the basin taken in the $(y, z)$ plane. The projection of the target points onto the plane is shown to indicate the relative size of the target. Axis are in metres.

Figures 4-5 show a typical simulated trajectory for the closed-loop system. The evolution of the image features in the image plan and the local and slight increase of the error $\delta_{1}$ are respectively shown in Figures 6 and 7 .

\footnotetext{
3 According to standard aeronautical conventions height is measured down relative to the aircraft and hence the height of the $\mathrm{X} 4$-flyer is negative with respect to the world frame.
}

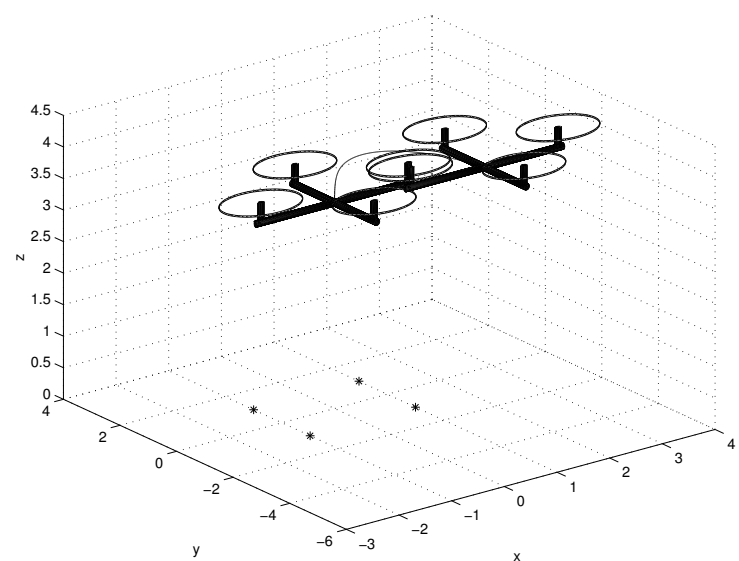

Fig. 4. Positioning of the X4-flyer with respect to the target. Initial condition within the guaranteed basin of attraction.
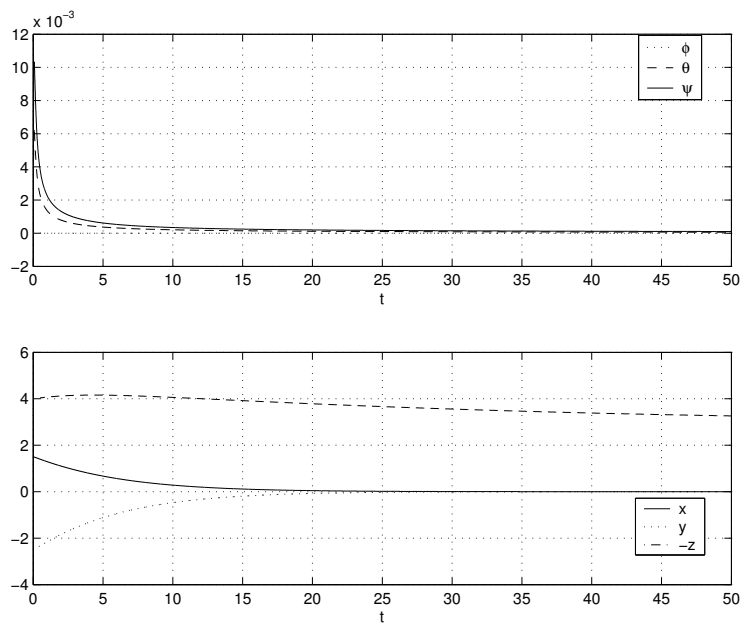

Fig. 5. Evolution of the attitude and position of the X4-flyer in 'roll' (theta), 'pitch' (psi) and 'yaw' (phi) Euler angles and Cartesian space coordinates $(x, y$, and $z)$. Initial condition within the guaranteed basin of attraction.

\section{Conclusion}

This paper has proposed a 2D image-based strategy for visual servo control of a class of under-actuated dynamic systems. It extends previous work by the authors [6] by avoiding the use of inertial information in the definition of the visual error. A decoupled control design is undertaken followed by a fully coupled stability and robustness analysis. The simulation of the control of an X4flyer indicates that the control will function effectively in practice.

\section{Acknowledgements}

This work is partly supported by Australian Research Council Grant DP0342849. 


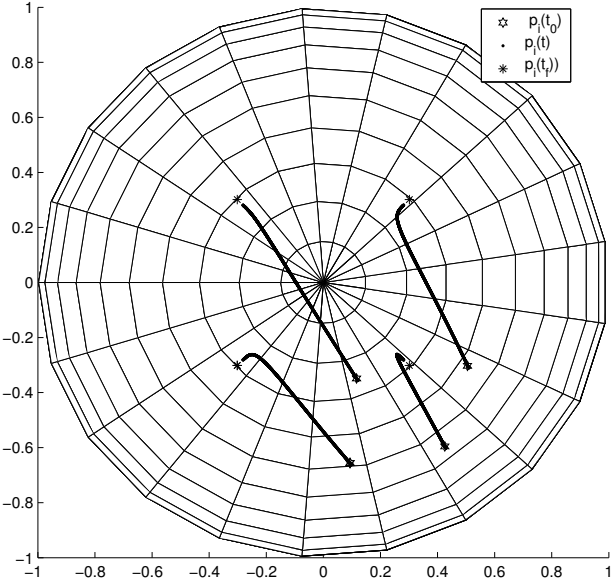

Fig. 6. Evolution of the image features in the image plan. Initial condition within the guaranteed basin of attraction.

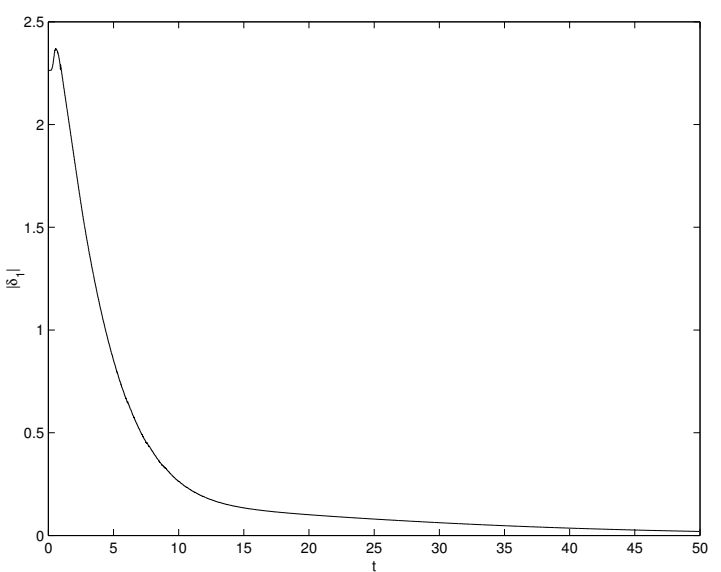

Fig. 7. Evolution of the norm of the image error $\delta_{1}$. Initial condition within the guaranteed basin of attraction.

\section{References}

[1] E. Altug, J. Ostrowski, and R. Mahony. Control of a quadrotor helicopter using visual feedback. In Proceedings of the IEEE International Conference on Robotics and Automation, ICRA2002, Washington DC, Virginia, USA, 2002.

[2] A. Astolfi, L. Hsu, M. Netto, and R. Ortega. Two solutions to the adaptive visual servoing problem. IEEE transactions on Robotics and Automation, 18(3):387-392, 2002.

[3] M. Dahlen E. Frazzoli and E. Feron. Trajectory tracking control design for autonomous helicopters using a backstepping algorithm. In Proceedings of the American Control Conference ACC2000, pages 4102-4107, Chicago, Illinois, USA, 2000.

[4] B. Espiau, F. Chaumette, and P. Rives. A new approach to visual servoing in robotics. IEEE Transactions on Robotics and Automation, 8(3):313-326, 1992.

[5] E. Frazzoli, M. A. Dahleh, and E. Feron. Real-time motion planning for agile autonomous vehicles. AIAA Journal of Guidance, Control, and Dynamics, 5(1):116-129, 2002.

[6] T. Hamel and R. Mahony. Visual servoing of an underactuated dynamic rigid-body system: An image based approach. IEEE Transactions on Robotics and Automation, 18(2):187-198, April 2002.

[7] T. Hamel, R. Mahony, R. Lozano, and J. Ostrowski. Dynamic modelling and configuration stabilization for an X4-flyer. In International Federation of Automatic Control Symposium, IFAC2002, Barcelona, Spain, 2002.

[8] S. Hutchinson, G. Hager, and P. Cork. A tutorial on visual servo control. IEEE Transactions on Robotics and Automation, 12(5):651-670, 1996.

[9] R. Kelly. Robust asymptotically stable visual servoing of planar robots. IEEE Transactions on Robotics and Automation, 12(5):759-766, 1996.

[10] H. K. Khalil. Nonlinear Systems. Prentice Hall, New Jersey, U.S.A., second edition, 1996.

[11] M. Krstic, I. Kanellakopoulos, and P. V. Kokotoviv. Nonlinear and adaptive control design. American Mathematical Society, Rhode Islande, USA, 1995.

[12] Y. Ma, J. Kosecka, and S. Sastry. Vision guided navigation for a nonholonomic mobile robot. IEEE Transactions on Robotics and Automation, 15(3):521-536, 1999.

[13] R. Mahony and T. Hamel. Robust trajectory tracking for a scale model autonomous helicopter. International Journal on Robust and Nonlinear Control, 14(12):1035-1059, August 2004.

[14] R. Mahony, T. Hamel, and F. Chaumette. A decoupled image space approach to visual servoing for a robotic manipulator. In Proceedings of the International Conference on Robotics and Automation, ICRA2002, Washington DC, Virginia, USA, 2002.

[15] J. Roberts, P. Corkes, and G. Buskey. Low-cost flight control system for a small autonomous helicopter. In Proceedings of the Australasian Conference on Robotics and Automation, ACRA02, Auckland, New-Zealand, 2002.

[16] O. Shakernia, Y. Ma, T. J. Koo, and S. Sastry. Landing an unmanned air vehicle: vision based motion estimation and nonlinear control. Asian Journal of Control, 1(3):128-146, 1999.

[17] Yantao Shen, Dong Sun, Yun-Hui Liu, and Kejie Li. Asymptotic trajectory tracking of manipulators using uncalibrated visual feedback. IEEE/ASME Trasnactions on Mechatronics, 8(1):87-98, 2003.

[18] E. Zergeroglu, D. Dawson, M. de Queiroz, and S. Nagarkatti. Robust visual-servo control of robot manipulators in the presence of uncertainty. In Proceedings of the 38th Conference on Decision and Control, CDC1999, Phoenix, Arizona, USA. 1999. 